\title{
Comparison of the Long-term Outcomes of Combined Phacovitrectomy and Sequential Surgeries for Macular Hole and Cataract
}

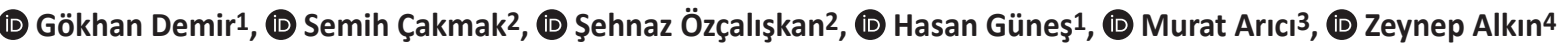 \\ ${ }^{1}$ Fatih Sultan Mehmet Training and Research Hospital, Clinic of Ophthalmology, Istanbul, Turkey \\ ${ }^{2}$ Beyoğlu Eye Training and Research Hospital, Clinic of Ophthalmology, Istanbul, Turkey \\ ${ }^{3}$ Tunceli State Hospital, Clinic of Ophthalmology, Tunceli, Turkey \\ ${ }^{4}$ Etiler Dünyagöz Hospital, Clinic of Ophthalmology, Istanbul, Turkey
}

\section{Abstract}

Objective: This study aimed to compare the long-term functional and anatomical outcomes of combined phacovitrectomy and sequential vitrectomy and cataract surgery for macular hole (MH) and cataract.

Methods: This retrospective comparative study analyzed the medical records of 58 patients who underwent phaco vitrectomy ( $\mathrm{n}=24)$ or sequential surgery $(n=34)$ for MH and cataract between March 2014 and March 2016. Patients were divided into the combined surgery group, which underwent combined surgery for $\mathrm{MH}$ and cataract extraction, and the sequential surgery group, which underwent vitrectomy first, followed by cataract extraction. The primary outcome measures were the best-corrected visual acuity (BCVA) before surgery and at 3, 6 , 12 , and 24 months after surgery and successful hole closure.

Results: A total of 58 patients were included in this study. The change in BCVA at 3 months after surgery was significantly better in the combined surgery group than in the sequential surgery group. However, no significant difference was found between the groups at 6 , 12 , and 24 months after surgery. Cataract surgery was performed at $4.9 \pm 1.2$ months after the first surgery (vitrectomy) in the sequential surgery group. In the success group, the rates of hole closure were 95.8\% (23/24) and 97\% (33/34) in the combined and sequential surgery groups, respectively.

Conclusion: Combined phaco vitrectomy and sequential vitrectomy and cataract surgery are safe and effective methods for treatment of $\mathrm{MH}$ and cataract. Although both methods demonstrate similar anatomical and functional success, combined surgery appears superior over sequential surgery because of the early visual improvement, decreased patient morbidity, and lower cost.

Keywords: Macular hole surgery, closure success, cataract

\section{INTRODUCTION}

In recent years, treatment of a macular hole $(\mathrm{MH})$ has shown considerable improvement with the use of optical coherence tomography (OCT) in clinical practice. OCT technology was the first to demonstrate that a vitreoretinal surgery (VRS) may improve visual acuity in $\mathrm{MH}(1)$. Moreover, advancements in surgical techniques and instrumentations lead to more favorable functional and anatomical outcomes in $\mathrm{MH}(2)$.

$\mathrm{MH}$ commonly occurs in the elderly population. In cases with coexisting cataract, VRS may be more challenging due to poor surgical view secondary to media opacities. Combined phacovitrectomy is an approved treatment option in patients 
with combined cataract and vitreoretinal disorders $(3,4)$. This approach improves the surgical view, increases postoperative visual rehabilitation, decreases the need for a secondary procedure, and reduces cost.

In addition, cataract development is common in phakic eyes after vitrectomy $(5,6)$. Undesirable physiological effects of irrigation solutions, accidental mechanical injury, or prolonged gas exposure may accelerate cataract formation after surgery (7). In cataract surgery, more accurate and reliable postoperative refractive outcomes were obtained due to the developments in surgical technique, intraocular lens (IOL) formulations, and use of premium IOLs (8). However, cataract surgery is more complicated in vitrectomized eyes. Anatomical changes such as the absence of vitreous support, deeper anterior chamber, unstable lens capsules, posterior capsular plaques, and weakened zonules are the main risk factors for intraoperative complications (9-14).

Previous studies have described anatomical and functional results of both combined and sequential surgeries in $\mathrm{MH}$ (15). However, the effect of lens status during surgery on surgical outcomes is still unknown. Thus, this study aimed to compare the long-term functional and anatomical outcomes of combined phacovitrectomy and sequential vitrectomy and cataract surgeries for eyes with coexisting $\mathrm{MH}$ and cataract.

\section{METHODS}

\section{Study Population}

For this retrospective comparative study, we reviewed the charts of patients with both $\mathrm{MH}$ and cataract treated between March 2014 and March 2016. This study adheres to the tenets of the Declaration of Helsinki, and patients gave their written permission before surgery. The study was approved by Beyoğlu Institutional Review Board (date: 31/10/2018- decision number: 19/C-1).

This study included 58 patients, with a follow-up duration of at least 24 months. The patients were divided into two groups according to the timing of vitrectomy and cataract surgery: the combined surgery group consists of patients who underwent phacovitrectomy, and the sequential surgery group consists of patients who underwent vitrectomy, followed by cataract surgery. Medical records of each patient were reviewed, and the following data were recorded: age, sex, systemic diseases, followup duration, coexisting retinal diseases, intraocular tamponade, intraoperative and postoperative complications [such as intraocular pressure (IOP) elevation, reactions or synechia in the anterior chamber, hyphema, and retinal tears or detachment], and systemic and topical medications. Patients had undergone complete ophthalmologic evaluation, including assessment of visual acuity, IOP measurement using Goldmann applanation tonometry, slit-lamp biomicroscopy, fundoscopy, and OCT before surgery and at 1 day, 1 week, and 1, 3, 6, 12, and 24 months after surgery. At baseline and follow-ups, macular structural assessment was made using Spectralis (Heidelberg Engineering, Heidelberg, Germany). Then, 25-line and 30-degree scans were performed, and the fovea was determined as the center. Gass staging was done based on the preoperative OCT images, and the hole base diameter, maximum hole height, and shortest diameter of the hole opening were calculated. In each patient, preoperative peripheral retinal examination was performed using wide-field lens. The main outcome measure was the bestcorrected visual acuity (BCVA) before surgery and at 3, 6, 12, and 24 months after surgery, IOP changes, use of antiglaucomatous medications, and hole closure rates during postoperative visits. Secondary outcome measures were intraoperative and postoperative complications.

\section{Inclusion and Exclusion Criteria}

The inclusion criteria were as follows: patients who underwent vitrectomy for idiopathic MH between March 2014 and March 2016 and patients with follow-up duration of at least 24 months. The exclusion criteria for all participants were as follows: Any history of refractive or intraocular surgery, history of blunt and penetrating ocular trauma, traumatic $\mathrm{MH}$, retinal detachment associated with $\mathrm{MH}$, uveitis, diabetic retinopathy, glaucoma, age-related macular degeneration, and high myopia (i.e., greater than six diopters).

\section{Surgical Technique}

Surgical decisions were made after discussing with the patients the risks and benefits of surgery. As preparation, the periocular skin (with 10\% concentration) and conjunctival fornix (with 5\% concentration) were wiped with povidone-iodine. All patients received peribulbar anesthesia $10 \mathrm{~min}$ before surgery, induced by subtenon injection of $2 \mathrm{~mL}$ of lidocaine hydrochloride $20 \mathrm{mg} /$ $\mathrm{mL}+$ epinephrine $0.0125 \mathrm{mg} / \mathrm{mL}$ combined with hyaluronidase $300 \mathrm{IU}$. In the combined surgery group, cataract surgery was performed through two corneal incisions: a main incision of 2.6 $\mathrm{mm}$ and a side port incision of $0.9 \mathrm{~mm}$. The phacoemulsification method was used for cataract extraction. Foldable, single-piece posterior chamber IOLs (IOL; Zaracom Monofocal 1-piece IOL) were implanted in the bag in most of the patients. However, three-piece IOLs were implanted in the sulcus if needed. A single corneal suture (Ethicon 10-0; Ethicon Inc., San Angelo, TX) was applied to the corneal tunnel. Then, three-port pars plana 
vitrectomy was performed with the constellation system (Alcon Laboratories, Inc., Fort Worth, TX) using 23-gauge valved trocars, and the vitreous gel and posterior hyaloid were removed. The internal limiting membrane (ILM) was stained with a brilliant blue solution and peeled from an area within 2-3 disc diameters from the fovea using forceps. ILM peeling was performed starting from the temporal region to minimize the risk of damaging the papillomacular bundle. The retinal periphery was examined with a wide-angle imaging system after the procedure, and laser photocoagulation (Purepoint ${ }^{\circledR} 532 \mathrm{~nm}$ Laser) was performed if retinal tears were found. Following the liquid-air exchange, longer-acting gases (C3F8 or SF6) were used to buffer the hole. All surgeries have been performed by the same surgeon (Z.A).

In the sequential surgery group, $\mathrm{MH}$ surgery was performed following the same method, and cataract surgery was performed when the cataract caused visual impairment during follow-up. For each patient, intraoperative and postoperative complications were noted. All patients were advised to maintain a facedown position as much as possible 1 week after MH surgery. Standard anti-inflammatory, antibiotic, and, if necessary, antiglaucomatous treatments were prescribed within 1 month of surgery. In case of an anterior chamber reaction, an antiinflammatory drug was administered more often.

\section{Statistical Analysis}

All statistical analyses were done using SPSS Statistics version 25.0 for Windows (SPSS Inc., Chicago, IL, USA). Visual acuities were converted to logarithm of the minimal angle of resolution units.
Data normality was assessed using Kolmogorov-Smirnov test. Categorical variables were recorded as numbers and numerical variables as mean and standard deviation. Depending on the variable type, data were analyzed by Wilcoxon, Mann-Whitney $\mathrm{U}$, and Fisher exact tests. The $\mathrm{p}$ value $<0.05$ was accepted for statistical significance.

\section{RESULTS}

A total of 58 patients were included in this study, with 24 patients in the combined surgery group and 34 patients in the sequential surgery group. The mean patient age was $68.3 \pm 5.9$ and $67.5 \pm 6.2$ years in the combined and sequential surgery groups, respectively. Sex distribution was similar between two the groups $(p=0.76)$. The demographic characteristics of the patients are summarized in Table 1. The mean follow-up time was $26 \pm 2.4$ months. No significant difference was found in $\mathrm{MH}$ size and the period between the appearance of clinical findings and surgery in both groups $(p=0.24, p=0.45$, respectively). In the overall study group, 12 cases were in stage 2, 28 cases were in stage 3 , and 18 cases were in stage 4 according to the Gass classification. The mean interval between vitrectomy and cataract surgery was $4.9 \pm 1.2$ months in the sequential surgery group. The mean change in the BCVA at 3 month after surgery was statistically significantly better in the combined surgery group than in the sequential surgery group. No significant difference was noted between the two groups at 6,12 , and 24 months after surgery. Changes in the BCVA over time in the two groups are presented in Figure 1. The rates of hole closure in

\begin{tabular}{|c|c|c|c|}
\hline & Combined group $n=24$ & Consecutive group $n=34$ & $\mathrm{p}$ value \\
\hline Gender (M/F) & $11 / 13$ & $16 / 18$ & 0.76 \\
\hline Age (years)a & $68.3 \pm 5.9$ & $67.5 \pm 6.2$ & 0.81 \\
\hline Mean size of $\mathrm{MH}(\mu \mathrm{m})$ & $424 \pm 128$ & $431 \pm 138$ & 0.24 \\
\hline Duration of MH (months) & $5.2 \pm 1.3$ & $5.8 \pm 1.5$ & 0.45 \\
\hline Mean BCVA (LogMAR) & - & - & - \\
\hline Preoperative BCVA & $0.89 \pm 0.21$ & $0.87 \pm 0.24$ & 0.69 \\
\hline Postoperative BCVA at 3 rd months & $0.33 \pm 0.18$ & $0.69 \pm 0.16$ & $<0.05$ \\
\hline Postoperative BCVA at $6^{\text {th }}$ months & $0.30 \pm 0.17$ & $0.31 \pm 0.20$ & 0.75 \\
\hline Postoperative BCVA at $12^{\text {th }}$ months & $0.31 \pm 0.19$ & $0.32 \pm 0.21$ & 0.77 \\
\hline Postoperative BCVA at 24th months & $0.31 \pm 0.18$ & $0.31 \pm 0.18$ & 0.81 \\
\hline Mean BCVA change (LogMAR) & - & - & - \\
\hline At 3rd months after surgery & $0.56 \pm 0.20$ & $0.18 \pm 0.23$ & $<0.05$ \\
\hline At $6^{\text {th }}$ months after surgery & $0.59 \pm 0.19$ & $0.56 \pm 0.19$ & 0.55 \\
\hline At $12^{\text {th }}$ months after surgery & $0.58 \pm 0.21$ & $0.55 \pm 0.21$ & 0.61 \\
\hline At $24^{\text {th }}$ months after surgery & $0.58 \pm 0.20$ & $0.56 \pm 0.20$ & 0.58 \\
\hline
\end{tabular}


the overall study group, combined surgery group, and sequential surgery group were $96.5 \%$ (56/58), 95.8\% (23/24), and 97\% (33/34), respectively. During vitrectomy in the combined surgery group, three patients had retinal tears, and one patient developed retinal detachment. In the sequential study group, four patients had retinal tears, and retinal detachment was observed in one patient. Retinal tears were treated with endo-photocoagulation, and routine surgical procedures were performed to treat the detached retina.

During cataract surgery. Run-out capsulorhexis in two cases and posterior capsule rupture in two cases were noted among 24 patients in the combined surgery group. In the sequential surgery group, posterior capsule rupture $(n=4)$, run-out capsulorhexis $(n=3)$, and crystalline lens drop $(n=1)$ occurred during cataract surgery. In the overall study group, 10 patients (6 in the combined group and 4 in the sequential group) had IOP elevation, which responded to antiglaucomatous medication. However, no significant difference in IOP levels was found between the groups at 1, 3, 6, 12, and 24 months after surgery. As endotamponade, C3F8 gas was used in 20 (83.3\%) and 25 (73.5\%) cases and SF6 gas was used in 4 (16.7\%) and 9 (26.5\%) cases in the combined and sequential surgery groups, respectively.

\section{DISCUSSION}

As demonstrated in previous literature and in this study, combined phacovitrectomy and sequential vitrectomy and cataract surgery are both safe and effective methods in patients with coexisting cataract and $\mathrm{MH}$ (16). Some authors have attempted to describe the best surgical method in $\mathrm{MH}$ surgery to reach a closure rate higher than $90 \%(17,18)$. Cataract formation that causes decreased vision after successful VRS is commonly seen in $\mathrm{MH}$. Nuclear sclerosis following vitrectomy has been reported to occur in $75 \%-90 \%$ of patients with $\mathrm{MH}$

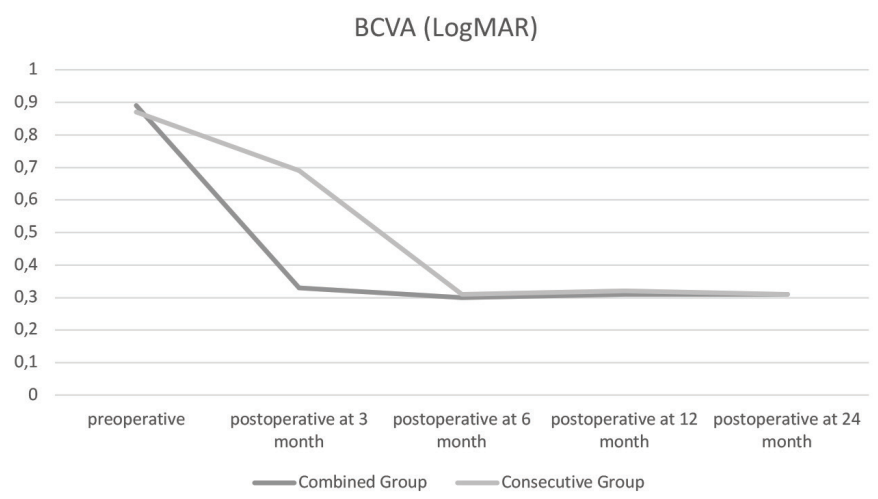

Figure 1. The graph shows the BCVA change of both groups over time BCVA: Best-corrected visual acuity, LogMAR: Logarithm of minimal angle of resolution
$(19,20)$. Lens opacities may cause difficulty in the visualization of the ILM during surgery. Thus, inadvertent trauma to the neurosensory retina may occur due to the excessive amount of force applied during ILM peeling (21). Combined phaco vitrectomy has many advantages for both the patient and surgeon $(22,23)$. The patient undergoing combined surgery will have fewer visits and will not experience perioperative stress for a second time. By contrast, cataract surgery in vitrectomized eyes is more challenging for surgeons. Zonular weakness, fragile posterior capsule, and unstable anterior chamber are factors that cause difficulties and complications during cataract surgery in vitrectomized eyes (23). In this study, a few complications, including runout capsulorhexis, posterior capsule rupture, and crystalline lens drop, were noted during cataract surgery in the sequential surgery group. Therefore, it is a good surgical option to combine vitrectomy with phacoemulsification in patients with coexisting cataract and $\mathrm{MH}$. Retinal imaging will be more convenient during surgery after cataract removal. However, surgeons should consider the possibility of corneal edema after phacoemulsification in combined surgeries, which impairs retinal visualization. In addition, some authors have emphasized that postoperative inflammation may be more common in combined surgeries, and complications such as posterior synechia, optic capture, and anterior chamber reaction are not rare (24). They have also noted that these postoperative findings might be minimized by using anti-inflammatory drugs and appropriate positioning. In this study, we did not observe these postoperative complications, except anterior chamber reaction in four patients in the combined surgery group.

In this study, functional and anatomical outcomes in both study groups were similar. The mean change in the BCVA at 3 months after surgery was significantly better in the combined surgery group than in the sequential surgery group. However, this significant difference was not observed in other study time points because the mean change in the BCVA after cataract surgery increased statistically significantly in the sequential surgery group and BCVA improvement was similar between the two groups at 6, 12,18, and 24 months after surgery.

\section{Study Limitations}

The limitations of our study are its retrospective design and small sample size. The lack of data with regard to preoperative cataract grading is another limitation. A 24-month follow-up period is a strong aspect of the study. Prospective studies evaluating the effect of combined surgery on anterior segment structures are needed to clarify the corneal changes after surgery. 


\section{CONCLUSION}

Combined phacovitrectomy and sequential vitrectomy and cataract surgery are both safe and effective methods in the treatment of coexisting $\mathrm{MH}$ and cataract. Although both methods are similar in terms of anatomical and functional success, combined surgery has the advantages of early visual improvement, decreased patient morbidity, and reduced cost. In addition, combined surgery appears advantageous because more cataract surgery complications were encountered in the sequential surgery group.

\section{Ethics}

Ethics Committee Approval: The study was approved by Beyoğlu Institutional Review Board (date: 31/10/2018- decision number: 19/C-1).

Informed Consent: Informed consent was obtained from all individual participants included in the study.

Peer-review: Externally peer-reviewed.

\section{Authorship Contributions}

Surgical and Medical Practices: Z.A., Concept: G.D., Design: H.G., Data Collection or Processing: S.C.., S..Ö., Analysis or Interpretation: G.D., S.C.., M.A., Literature Search: G.D., Z.A., Writing: G.D., S.Ç.

Conflict of Interest: No conflict of interest was declared by the authors.

Financial Disclosure: The authors declared that this study received no financial support.

\section{REFERENCES}

1. Kelly NE, Wendel RT. Vitreous surgery for idiopathic macular holes. Results of a pilot study. Arch Ophthalmol 1991;109:654-9.

2. Scott IU, Moraczewski AL, Smiddy WE, Flynn HW Jr, Feuer WJ. Long-term anatomic and visual acuity outcomes after initial anatomic success with macular hole surgery. Am J Ophthalmol 2003;135:633-40.

3. Koenig SB, Mieler WF, Han DP, Abrams GW. Combined phacoemulsification, pars plana vitrectomy, and posterior chamber intraocular lens insertion. Arch Ophthalmol 1992;110:1101-4.

4. Demetriades AM, Gottsch JD, Thomsen R, Azab A, Stark WJ, Campochiaro $\mathrm{PA}$, et al. Combined phacoemulsification, intraocular lens implantation, and vitrectomy for eyes with coexisting cataract and vitreoretinal pathology. Am J Ophthalmol 2003;135:291-6.

5. Thompson JT, Glaser BM, Sjaarda RN, Murphy RP. Progression of nuclear sclerosis and long-term visual results of vitrectomy with transforming growth factor beta-2 for macular holes. Am J Ophthalmol 1995;119:48-54.

6. Chung TY, Chung H, Lee JH. Combined surgery and sequential surgery comprising phacoemulsification, pars plana vitrectomy, and intraocular lens implantation: comparison of clinical outcomes. J Cataract Refract Surg 2002;28:2001-5.
7. D'Amico DJ. Vitreoretinal surgery principles and application. In: Albert DM, Jacobiec FA (eds). Principles and Practice of Ophthalmology. Vol. 2. Philadelphia:WB Saunders. 1994;1133-8.

8. Ladas JG, Stark WJ. Improving cataract surgery refractive outcomes. Ophthalmology 2011;118:1699-700.

9. Lacalle VD, Gárate FJO, Alday NM, Garrido JAL, Agesta JA. Phacoemulsification cataract surgery in vitrectomized eyes. J Cataract Refract Surg 1998;24:806-9.

10. McDermott ML, Puklin JE, Abrams GW, Eliott D. Phacoemulsification for cataract following pars plana vitrectomy. Ophthalmic Surg Lasers 1997;28:558-64.

11. Pinter SM, Sugar A. Phacoemulsification in eyes with past pars plana vitrectomy: case control study. J Cataract Refract Surg 1999;25:556-61.

12. Grusha YO, Masket S, Miller KM. Phacoemulsification and lens implantation after pars plana vitrectomy. Ophthalmology 1998;105:28794.

13. Sneed S, Parrish RK, Mandelbaum S, O'Grady G. Technical problems of extracapsular cataract extraction after vitrectomy. Arch Ophthalmol 1986;104:1126-7.

14. Smiddy WE, Stark WJ, Michels RG Maumenee AE, Terry AC, Glaser BM. Cataract extraction after vitrectomy. Ophthalmology 1987;94:483-7.

15. Muselier A, Dugas B, Burelle X, Passemard M, Hubert I, Mathieu B, et al. Macular hole surgery and cataract extraction: combined vs consecutive surgery. Am J Ophthalmol 2010;150:387-91.

16. Theocharis IP, Alexandridou A, Gili NJ, Tomic Z. Combined phacoemulsification and pars plana vitrectomy for macular hole treatment. Acta Ophthalmol Scand 2005;83:172-5.

17. Park DW, Sipperley JO, Sneed SR, Dugel PU, Jacobsen J. Macular hole surgery with internal-limiting membrane peeling and intravitreous air. Ophthalmology 1999;106:1392-7.

18. Passemard M, Yakoubi Y, Muselier A, Hubert I, Guillaubey A, Bronet AM, et al. Long-term outcome of idiopathic macular hole surgery. Am J Ophthalmol 2010;149:120-6.

19. Lahey JM, Francis RR, Fong DS, Kearney JJ, Tanaka S. Combining phacoemulsification with vitrectomy for treat- ment of macular holes. Br J Ophthalmol 2002;86:876-8.

20. Freeman WR, Azen SP, Kim JW, el-Haig W, Mishell DR 3rd, Bailey I. Vitrectomy for the treatment of full-thickness stage 3 or 4 macular holes. Results of a multicentered randomized clinical trial. The Vitrectomy for Treatment of Macular Hole Study Group. Arch Ophthalmol 1997;115:1121.

21. Kumagai K, Furukawa M, Ogino N, Larson E. Incidence and factors related to macular hole reopening. Am J Ophthalmol 2010;149:127-32.

22. Grusha YO, Masket S, Miller KM. Phacoemulsification and lens implantation after pars plana vitrectomy. Ophthalmology 1998;105:28794.

23. Treumer F, Bunse A, Rudolf M, Roider J. Pars plana vitrectomy, phacoemulsification and intraocular lens implantation. Comparison of clinical complications in a combined versus two-step surgical approach. Graefes Arch Clin Exp Ophthalmol 2006;244:808-15.

24. Kotecha AV, Sinclair SH, Gupta AK, Tipperman R. Pars plana vitrectomy for macular holes combined with cataract extraction and lens implantation. Ophthalmic Surg Lasers 2000;31:387-93. 